\title{
Dipole Moments of the Bioactive Constituents Present in Flutab Drug by Ab-Initio Calculations
}

\author{
Raghad Alajlani, Amal Alssadi \\ Faculty of Science, Chemistry Department, King Abdulaziz University, Jeddah, Saudi Arabia \\ Email: nwazzan@kau.edu.sa
}

How to cite this paper: Alajlani, R. and Alssadi, A. (2019) Dipole Moments of the Bioactive Constituents Present in Flutab Drug by Ab-Initio Calculations. Open Journal of Physical Chemistry, 9, 216-220. https://doi.org/10.4236/ojpc.2019.94013

Received: September 30, 2019

Accepted: November 5, 2019

Published: November 8, 2019

Copyright $\odot 2019$ by author(s) and Scientific Research Publishing Inc. This work is licensed under the Creative Commons Attribution International License (CC BY 4.0).

http://creativecommons.org/licenses/by/4.0/

\begin{abstract}
Many of the technology and computational chemistry applications are used to study drugs and their biological effects. Flutab ${ }^{\circledR}$ drug contains Paracetamol, Diphenhydramine and Pseudoephedrine. Ab-initio calculations were performed at DFT/B3LYP and HF methods with three basis sets, namely, STO-3G, 3-21G, and 6-31G(d) in order to calculate the dipole moments of the three constituents of Flutab ${ }^{\oplus}$ drug. The Diphenhydramine compound was found to be the most stable constituent, with the lowest value of dipole moment.
\end{abstract}

\section{Keywords}

Flutab $^{\circledR}$, DFT Calculations, HF Calculations, Dipole Moment

\section{Introduction}

Flutab $^{\bowtie}$ drug (generic name: Pseudoeph-DM-GG-Acetaminophen) is a pain reliever medication to remove cold symptoms, see Figure 1. This combination medication is used to temporarily treat cough, chest congestion, fever, body aches, and stuffy nose symptoms caused by the common cold, flu, or other breathing illnesses (e.g., sinusitis, bronchitis).

Each tablet of Flutab ${ }^{\star}$ contains: Paracetamol $(500 \mathrm{mg})$, Pseudoephedrine hydrochloride (30 mg) and Diphenhydramine hydrochloride (25 mg) [1] [2]. Flu$\mathrm{tab}^{\circledR}$ is dependent on the presence of pain-relieving Paracetamol. Pseudoephedrine helps relieve congestion and Diphenhydramine as anti-histamine constituent. The three bioactive compounds Paracetamol, Pseudoephedrine, and Diphenhydramine are abbreviations for N-(4-hydroxyphenyl)acetamide, 2-(benzhydryloxy)-N,N-dimethylethanamine, and

(1S,2S)-2-(methylamino)-1-phenylpropan-1-ol, respectively. The chemical 


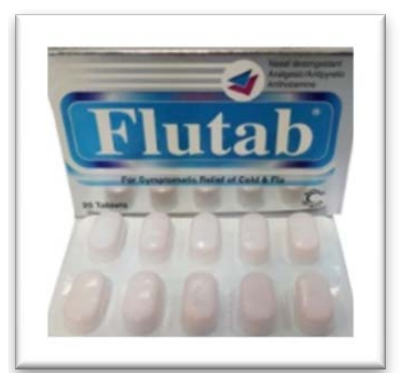

Figure 1. Flutab ${ }^{\otimes}$ drug package.

structures of the Flutab ${ }^{\otimes}$ bioactive constituents along with their IUPAC names and abbreviation are shown in Figure 2.

Paracetamol is a pain reliever and a fever reducer. The exact mechanism of action of is not known. Paracetamol is used to treat many conditions such as headache, muscle aches, arthritis, backache, toothaches, colds, and fevers. It relieves pain in mild arthritis but has no effect on the underlying inflammation and swelling of the joint. Pseudoephedrine is a sympathomimetic drug of the phenethylamine and amphetamine chemical classes. It may be used as a nasal/sinus decongestant, as a stimulant, or as a wakefulness-promoting agent in higher doses. Diphenhydramine is an antihistamine mainly used to treat allergies. It is also used for insomnia, symptoms of the common cold, tremor in parkinsonism, and nausea. It is used by mouth, injection into a vein, and injection into a muscle.

Much of the technology and computational chemistry applications are used to study drugs and their biological effects. The successful applications of quantum chemical calculations included density functional theory (DFT), its time-dependent functional (TDDFT) and molecular dynamic simulations in studying the drugs behavior [3] [4], and delivering [5] were reported in large amounts of work. The aim of the study is to determine which of the bioactive compounds presented in Flutab ${ }^{\oplus}$ drug has more stability by calculations of the dipole moments of each constituent using DFT/B3LYP [6] and Hartree-Fock (HF) [7] methods. The GaussView program (version 5.0.8) [8] was used to draw the structures of the molecules. Dipole moments of these three compounds were determined using Gaussian 09 suite program [9].

\section{Ab-Initio Details}

The dipole moments of the three bioactive compounds were determined using Gaussian 09 software [9] and GaussView 5.0 program [8]. Gaussian 09 is an electronic structure package capable of predicting many properties of atoms, molecules, and reactive systems. It is a general-purpose computational chemistry software package initially released in 1970 by John Pople and his research group at Carnegie Mellon University as Gaussian. The name originates from Pople's use of Gaussian orbitals to speed up molecular electronic structure calculations as opposed to using Slater-type orbitals, a choice made to improve performance 
<smiles>CC(=O)Nc1ccc(O)cc1</smiles>

N-(4hydroxyphenyl)acetam ide (Paracetamol)

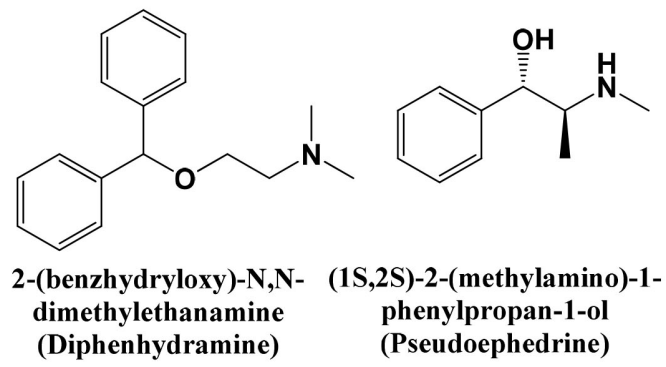

Figure 2. The chemical structures of Flutab ${ }^{\circledast}$ bioactive constituents along with their IUPAC names.

on the limited computing capacities of then-current computer hardware for Hartree-Fock calculations. GaussView a very advanced and powerful graphical user interface for Gaussian. With the aid of GaussView the molecule can be built, saved or loaded. GaussView can be used to start, monitor and control Gaussian calculations, Also, GaussView can be used to visualize and analyze different parameters. The optimizations were carried out at DFT with one of hybrid functionals, i.e., B3LYP, which stands for "Becke, 3-parameter, Lee-Yang-Parr". Other optimizations were carried out at the HF method. Three basis sets were used, the STO-3G (a minimal basis set, where 3 primitive Gaussian orbitals are fitted to a single Slater-type orbital (STO)). Also, two split-valence double-zeta basis sets. i.e., 3-21 G and 6-31 G(d). The two split-valence double-zeta basis sets are differing in the number of primitive Gaussians comprising each core and valence atomic orbital basis function, also, an additional polarization function is added on heavy atoms in $6-31 \mathrm{G}(\mathrm{d})$ denoted by (d). The frequency calculations were performed on all optimized geometries in order to confirm that these geometries are corresponding to minima points on the potential energy surface with the absence of any imaginary frequencies.

The optimized geometries of the three investigated compounds at the B3LYP/6-31G(d) level of theory as representative examples are shown in Figure 3 along with arrows indicating the direction of dipole moment vector. The dipole moments of the three investigated compounds at the DFT at B3LYP and HF methods with three basis sets, namely, STO-3G, 3-21 G, and 6-31 G(d) are listed in Table 1.

\section{Results and Discussion}

The obtained data in Table 1 shows that the values of dipole moment at the HF method are larger than those at the B3LYP functional with the STO-3G and 3-21 $\mathrm{G}$ basis sets. While, the obtained dipole moments at the B3LYP functional with 6-31 G(d) basis set are higher than those obtained at the HF method. This result indicated the effect of the type of basis set on such calculations. For each compound at the two methods, the dipole moment obtained with STO-3G basis set is the smallest, while the one obtained with $6-31 \mathrm{G}(\mathrm{d})$ is the largest, the obtained value with $3-21 \mathrm{G}$ basis set is an intermediate value. 


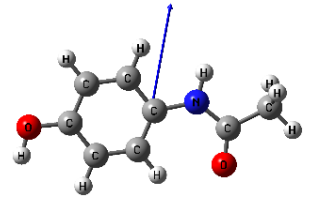

(a)

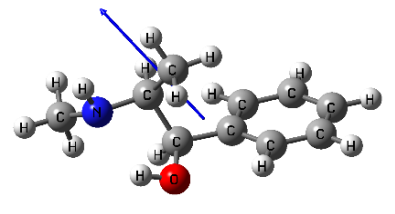

(b)

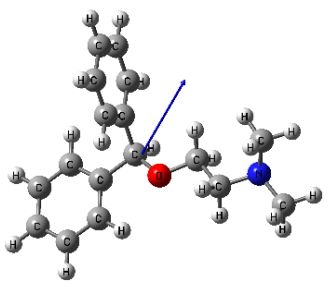

(c)

Figure 3. The geometries of the three bioactive constituents of Flutab ${ }^{\oplus}$ (a) Paracetamol. (b) Pseudoephedrine, and (c) Diphenhydramine as optimized at the DFT/B3LYP/6-31G(d) level of theory.

Table 1. Total Dipole moments (in Debye) of the three investigated compounds as calculated at the DFT/B3LYP and HF level of theory.

\begin{tabular}{ccccccc}
\hline & \multicolumn{3}{c}{ DFT/B3LYP } & \multicolumn{3}{c}{ HF } \\
\cline { 2 - 7 } Compound name & STO-3G & $3-21 \mathrm{G}$ & $6-31 \mathrm{G}(\mathrm{d})$ & STO-3G & $3-21 \mathrm{G}$ & $6-31 \mathrm{G}(\mathrm{d})$ \\
\hline Paracetamol & 1.4632 & 2.0904 & 2.1131 & 1.8224 & 2.5928 & 2.4109 \\
Diphenhydramine & 1.0410 & 1.3651 & 1.0863 & 1.0773 & 1.4069 & 1.0533 \\
Pseudoephedrine & 3.4684 & 3.4946 & 2.9005 & 2.6890 & 3.2765 & 2.8123 \\
\hline
\end{tabular}

However, the two methods with three basis sets show that the order of increasing the dipole moments values of the three compounds is: Diphenhydramine < Paracetamol < Pseudoephedrine. For instance, the dipole moments are 1.0863, 2.1131, and 2.9005 Debye for Diphenhydramine, Paracetamol, and Pseudoephedrine, respectively, at the B3LYP/6-31G(d) level of theory. If the dipole moment value is low, the compound will be more stable, and will be expected to be responsible for the bioactivity of Flutab ${ }^{\circledR}$ drug [2]. Therefore, according to the present result, the Diphenhydramine constituent is expected to be the most active constituent and will be responsible for the bioactivity of Flutab ${ }^{\circledast}$ drug.

\section{Conclusions}

The stability of compound is determined by calculating the dipole moment; if the dipole moment is low, stability will be more. Dipole moment calculations for Paracetamol, Diphenhydramine and Pseudoephedrine using DFT/B3LYP and HF methods with STO-3G, 3-21G, 6-31G(d) basis sets, indicated that Diphenhydramine was the most stable compound compared to other compounds in Flutab $^{\circledast}$, and expected that it is the most active one and it is responsible for the bioactivity of Flutab ${ }^{\circledR}$ drug.

\section{Acknowledgements}

The authors thank Dr. Nuha Wazzan for her supervision during this course which was a requirement for our bachelor's degree. 


\section{Conflicts of Interest}

The authors declare no conflicts of interest regarding the publication of this paper.

\section{References}

[1] Abu-Sehly, A.A. and Elabbar, A.A. (2011) Effect of Long-Term Physical Aging on the Kinetic Parameters in a Common Pharmaceutical Drug: Flutab ${ }^{\oplus}$. Physica B: Condensed Matter, 406, 319-323. https://doi.org/10.1016/j.physb.2010.10.032

[2] Suganya, M., Indumathy, L. and Sunmathi, S. (2018) In Silico Calculations of Dipole mOment by DFT and Drug Activity Predictions for the Bioactive Constituent Present in Coleus Aromatics. International Research Journal of Engineering and Technology (IRJET), 5, 268-273.

[3] Ghasemi, A.S., Mashhadban, F. and Ravari, F. (2018) A DFT Study of Penicillamine Adsorption over Pure and Al-Doped C-60 Fullerene. Adsorption-Journal of the International Adsorption Society, 24, 471-480. https://doi.org/10.1007/s10450-018-9960-3

[4] Ghasemi, A.S., et al. (2019) Adsorption Behavior of Metformin Drug on Boron Nitride Fullerenes: Thermodynamics and DFT Studies. Journal of Molecular Liquids, 275, 955-967. https://doi.org/10.1016/j.molliq.2018.11.124

[5] Hazrati, M.K., Javanshir, Z. and Bagheri, Z. (2017) B24N24 Fullerene as a Carrier for 5-Fluorouracil Anti-Cancer Drug Delivery: DFT Studies. Journal of Molecular Graphics and Modelling, 77, 17-24. https://doi.org/10.1016/j.jmgm.2017.08.003

[6] Becke, A.D. (1993) Density-Functional Thermochemistry. III. The Role of Exact Exchange. The Journal of Chemical Physics, 98, 5648-5652. https://doi.org/10.1063/1.464913

[7] Roothaan, C.C.J. (1951) New Developments in Molecular Orbital Theory. Reviews of Modern Physics, 23, 69-89. https://doi.org/10.1103/RevModPhys.23.69

[8] Dennington, R., Keith, T. and Millam, J. (2009) GaussView. S. Mission, Editor., Semichem Inc., Shawnee Mission, KS.

[9] Frisch, M.J. (2009) Gaussian 09 Programmer’s Reference. Gaussian. 